\title{
Philosophiques
}

\section{Les idéalistes britanniques et la poésie}

\section{W. J. Mander}

Volume 36, numéro 1, printemps 2009

L'idéalisme britannique

URI : https://id.erudit.org/iderudit/038009ar

DOI : https://doi.org/10.7202/038009ar

Aller au sommaire du numéro

Éditeur(s)

Société de philosophie du Québec

ISSN

0316-2923 (imprimé)

1492-1391 (numérique)

Découvrir la revue

Citer cet article

Mander, W. J. (2009). Les idéalistes britanniques et la poésie. Philosophiques, 36(1), 35-52. https://doi.org/10.7202/038009ar
Résumé de l'article

Cet article explore la conception que les idéalistes britanniques se firent de la relation entre la philosophie et la poésie. J'examine la classification proposée par Hegel ainsi que la façon dont ils la modifièrent, et les difficultés auxquelles ils firent face dans leur tentative d'accommoder les critiques bien connues de Platon. J'examine ensuite certaines critiques adressées aux idéalistes à partir du point de vue de la philosophie analytique pour en conclure qu'elles ne sont guère convaincantes. 


\title{
Les idéalistes britanniques et la poésie
}

\author{
W. J. MANDER \\ Harris Manchester College, Oxford
}

\begin{abstract}
RÉSUMÉ. - Cet article explore la conception que les idéalistes britanniques se firent de la relation entre la philosophie et la poésie. J'examine la classification proposée par Hegel ainsi que la façon dont ils la modifièrent, et les difficultés auxquelles ils firent face dans leur tentative d'accommoder les critiques bien connues de Platon. J'examine ensuite certaines critiques adressées aux idéalistes à partir du point de vue de la philosophie analytique pour en conclure qu'elles ne sont guère convaincantes.
\end{abstract}

\begin{abstract}
This article explores the relation between philosophy and poetry according to the British Idealists. I examine their place in Hegel's classification as they modified it and the difficulties that they faced when trying to accommodate Plato's well-known criticisms. I examine further some of the criticisms of the British Idealist conceptions from the standpoint of Analytic Philosophy, in order to show how inconclusive they are.
\end{abstract}

On ne peut pas comprendre la philosophie des idéalistes britanniques, dans toute sa diversité, sans comprendre au préalable le type d'activité qu'ils pensaient avoir entreprise, c'est-à-dire leur propre conception de la philosophie. Mais on ne peut pas comprendre totalement leur conception de la philosophie sans comprendre leur conception de la relation de la philosophie à la poésie, parce qu'ils concevaient ces activités comme étant étroitement corrélées ${ }^{1}$.

Il y a, bien sûr, deux concepts en jeu, et concevoir les deux en étroite relation nous en dit autant sur leur conception de la poésie que sur leur conception de la philosophie. Mais comme il ne s'agit pas ici d'un essai en théorie littéraire, mon intérêt se portera sur le second concept - sur ce que cette relation nous enseigne sur leur approche de la philosophie. À une époque

1. Cet énoncé soulève trois objections possibles, toutes équivalentes, dans une certaine mesure. 1) À cette époque, tout un chacun considérait la poésie avec sérieux. La société victorienne avait une estime pour la poésie que nous ne possédons plus aujourd'hui, et on pouvait citer des poèmes dans tout type d'écrits. Cependant, il est bien vrai de dire que les idéalistes ont porté cette estime à ses plus hauts sommets. 2) Les idéalistes britanniques n'étaient pas les seuls intellectuels à croire à l'existence d'une étroite relation entre la poésie et la philosophie; Coleridge, Carlyle, John Stuart Mill, et Leslie Stephen (pour n'en nommer que quatre) ont tous noté d'importantes relations. Cela est également vrai, mais l'on peut dire, une fois de plus, que les idéalistes ont exploré ce lien plus profondément que les autres. 3) Ce ne sont pas tous les idéalistes qui ont mis la poésie sur un tel piédestal. On peut certes l'admettre. Bien que tous les idéalistes témoignent d'un savoir sans pareil et d'un amour pour la poésie, ce fut particulièrement Edward Caird et ses successeurs, Henry Jones, John Mackenzie, Andrew Seth PringlePattison et Lord Haldane qui l'examinèrent en détail. T. H. Green, J. E. M. McTaggart et J. H. Muirhead, semblent, d'autre part, relativement immunisés contre ses charmes. 
où les philosophes se considèrent rarement comme les amis des poètes, cet article propose un examen de cette conception révolue et vise à répondre aux critiques les plus naïves qui lui ont été objectées.

\section{L'affinité de la poésie et de la philosophie}

De nos jours, peu de philosophes ${ }^{2}$ définiraient leur travail par rapport à la poésie, mais pour les idéalistes il s'agissait d'une relation naturelle et importante dans la mesure où il existe selon eux une profonde convergence de but entre les fins poursuivies par ces deux activités. La poésie et la philosophie recherchent toutes deux la compréhension; elles appartiennent au domaine du savoir. Elles recherchent de surcroît le même type de vérité, à savoir dévoiler les principes les plus cachés, les plus profonds et les plus universels qui soustendent la pensée et la réalité. Malgré leurs nombreuses et indéniables différences, philosophes et poètes sont au fond chercheurs et porte-parole des mêmes choses ${ }^{3}$.

$\mathrm{Si}$ on recherche les sources d'une telle conception, on se retrouve alors immédiatement dans un de ces domaines où l'étiquette "anglo-hégélien » s'impose immédiatement, d'autant plus que le modèle dont il est question ici est celui de la célèbre théorie du développement de l'esprit (Geist) de Hegel.

Pour l'expliquer en deux mots, la poésie occupe pour Hegel une place assez spécifique dans cette séquence développementale. Le troisième et dernier stade du chemin triadique de l'Esprit allant du Subjectif à l'Objectif vers l'Absolu est lui-même caractérisé par une triade — art, religion, philosophie cela impliquant que chacun de ces trois sujets expriment le même message fondamental, bien que d'une manière progressivement supérieure. L'Idée que la philosophie énonce sous une forme explicitement rationnelle, la religion l'exprime seulement sous une moindre forme, imagée, alors que l'art ne peut pas l'énoncer du tout, mais simplement nous la présenter sous une forme concrète, matérielle, sensorielle. Hegel insiste sur la tendance de l'art à idéaliser; il se peut que ce que l'art représente provienne de la nature, mais c'est le rôle de l'artiste de mettre en évidence sa signification universelle, et cela introduit une progression au sein de l'art lui-même, à commencer par ses formes les plus concrètes, telles que l'architecture et la sculpture, progressant tandis que l'esprit se retire graduellement du monde des sens pour finalement devenir religion. Pour esquisser la triade culminante de ce mouvement, la musique est plus intime et subjective que la peinture, puisque le temps

2. Bien sûr, je limite mon propos au monde anglo-saxon où, comme on l'a récemment exposé (Eagleton, 2007, 5), les barrières entre philosophie et poésie sont maintenues avec vigilance.

3. «Le poète, comme le philosophe, recherche la vérité, et l'on peut même dire qu'il s'agit du même type de vérité » (Caird 1892c, 55); «Finalement, la philosophie ne fait qu'un avec la poésie [...] Leur but est le même» (Jones 1909, 10); «On peut alors dire que les genres de poésie les plus recherchés visent le même type de perspicacité que la philosophie requiert » (Mackenzie, 1917, 16). 
remplace l'espace, alors que la poésie, en faisant la synthèse de la peinture et de la musique, constitue l'idéalisation finale du sensuel. En poésie, le message devient complètement interne, le mot prononcé devient simplement un symbole pour la pensée ou l'image mentale silencieuse. C'est le plus haut stade de l'art, un échelon sous la religion ${ }^{4}$.

Laissant maintenant Hegel de côté, tentons d'expliquer de manière plus détaillée comment les idéalistes britanniques concevaient la poésie comme l'expression du même type de vérité que celle de la philosophie, ce qui est un sujet aux nombreuses facettes.

1) Pour commencer, le savoir que toutes deux cherchent à révéler serait caché sous la surface des choses. Il n'y a peut-être pas de distinction plus importante dans la pensée idéaliste que celle qui existe entre l'apparence et la réalité; les choses que nous tenons pour réelles (comme la matière, le temps et l'espace) s'avèrent ne pas l'être, et les distinctions que nous pensions absolues (comme celles entre le sujet et l'objet, entre différents sujets, entre le fini et l'infini) s'avèrent changeantes. Le sens commun et le langage ordinaire ne sont pas des guides vers la réalité ultime. Mais, de la même manière, il peut ne pas y avoir de poésie ordinaire du sens commun - celle-ci ne fait du moins pas partie de ce qu'ils nomment « la grande poésie» [higher poetry]. Toute chose simplement commune ou vulgaire «transforme immédiatement la poésie en prose par sa présence », dit Caird. Bien qu'elle nous apparaisse sous une réalité sensuelle et immédiate, la poésie ne procède ainsi que pour révéler des choses plus profondes, c'est pourquoi «la vérité poétique ne se trouve pas à la surface ". Bien qu'il se puisse que le poète, comme le scientifique, interagisse avec le monde empirique, il ne s'y abandonne pas, et «le poète, comme le philosophe, est en quête d'une vérité plus profonde que celle qui fait l'objet de la science » (Caird, 1892c, 58-59). Il se peut que le poète fasse usage du sensuel et du quotidien, mais seulement afin de nous permettre de voir au-delà.

2) L'un des aspects les plus significatifs de la conception idéaliste de la philosophie est l'universalité de sa vision. Pour les idéalistes, le domaine de la philosophie est le plus vaste; elle est "la tentative de comprendre l'univers non simplement par étapes ou par fragments, mais d'une manière ou d'une autre comme un tout » (Bradley, 1897, 1), elle est la recherche "d'une vision unifiée de la totalité des choses" (Bosanquet 1927, 25-26). Elle pourrait ici sembler différente de la poésie, celle-ci présentant sans aucun doute ce qui est spécifique et concret. Mais, après réflexion, la question doit être vue sous un autre angle, car la poésie ne se concentre jamais sur l'objet individuel ou l'événement lui-même,

4. Je réfère aux Cours d'esthétique (Hegel, 1995-7), traduits en anglais dans Hegel, 1905. Bien sûr, cela n'est qu'une esquisse des plus rudimentaires de la théorie de l'art de Hegel. Pour plus de détails, voir Desmond, 1986. 
sa présentation du particulier n'a de valeur que lorsqu'elle nous permet d'y reconnaître l'universel. Et nous apprécions le poème qui nous dévoile des vérités universelles, tout comme nous le faisons pour la philosophie ${ }^{5}$.

3) Si nous voulions un slogan pour cerner la philosophie idéaliste britannique, nous aurions peut-être à nous contenter de "l'identité relative " ou de «l'unité dans la diversité ». C'est une notion omniprésente, difficile, et qui a plusieurs facettes, digne d'une étude à part entière. Dans le cadre de ce texte, il suffit simplement de faire remarquer que les idéalistes la considérèrent comme une notion que la poésie exprime aussi le mieux. Elle unifie le sensuel et l'abstrait. Plusieurs éléments particuliers, images, etc., sont tous unis ensemble par une seule idée. Étant toujours à la recherche de la vie, elle combine organisme, unité et but, là où la science (par contraste) cherche à analyser en mécanismes dénués de vie. La philosophie et la poésie visent, dans les mots de Caird, à restaurer «l'harmonie brisée» de la vie; elles parlent à l'individu tout entier (Caird, 1892c, 191). En réfléchissant à la manière dont Wordsworth relie ses éléments, Caird indique que c'est précisément ce rôle synthétique qui « fait que la poésie est la contrepartie et le coadjuteur de la philosophie» (Caird, 1892b, 153).

4) Apparence et réalité, universalité, unité dans la diversité: le poète dit la même chose que le philosophe, ou plutôt - cela peut raisonnablement lui être objecté - la même chose que les philosophes idéalistes; c'est précisément la conclusion qu'ils ont tiré. Les idéalistes avaient le sentiment de dire simplement ce que les générations précédentes avaient dit. Selon eux, la meilleure poésie est celle qui nous livre un message idéaliste en accord avec le leur. Il faut citer ici Henry Jones, qui avance dans, A Faith that Enquires, que "l'idéalisme reçoit tout autant son inspiration de Wordsworth, Coleridge et de leurs confrères poètes, qu'il reçoit son problème spécifique de Kant» (Jones, 1922, 194-195), tandis qu'un chapitre de son livre, Idealism as a Practical Creed (Jones, 1909), est intitulé "L'idéalisme de Wordsworth et de Browning », où il explique précisément comment la poésie a donné naissance à l'idéalisme philosophique.

Mais Jones n'était pas le seul à penser ainsi. Nous trouvons aussi J. S. Mackenzie, qui discute, dans son Introduction to Social Philosophy de 1890, de la façon dont la poésie récente a enseigné l'idéalisme (Mackenzie, 1890, 376-377), tandis que Bosanquet suggère dans Identity and Value que la conception de la nature ou de l'univers dans l'espace-temps «interprété comme un système vivant » — c'est-à-dire à la manière des idéalistes — est précisément «la signification à l'origine de l'art et de la poésie» (Bosanquet, 1912, 358). Et si l'on s'écarte de la poésie pour se tourner un moment vers la littérature, l'influence de Carlyle ne peut guère être sous-estimée. Beaucoup d'idéalistes

5. La philosophie tente de «nous faire reconnaître comme principe universel de la réalité cet idéal que la poésie nous expose par des créations particulières» (Caird, 1892c, 63). 
considéraient qu'ils ne faisaient que reformuler l'idéalisme qu'ils avaient découvert la première fois en tant qu'étudiants, dans son roman de 1832, Sartor Resartus. Hilda Oakley, une figure mineure du mouvement idéaliste, qui est très peu lue, ne voyait pas cette anticipation comme accidentelle. Elle suggéra que l'esprit du monde (Weltgeist) doit être filtré par le caractère national et qu'il était peut-être inévitable que l'esprit qui avait produit la philosophie sur le continent produisît la poésie en Grande-Bretagne; Wordsworth était le Spinoza britannique (Oakley, 1923, 93-95).

\section{Différences entre Hegel et les idéalistes}

Avant de céder à la tentation d'évacuer les idéalistes britanniques comme de simples colporteurs de la théorie de la poésie de Hegel, il faut noter deux différences. Toutes deux concernent des questions d'ordonnancement.

Dans le sillage de Hegel, un grand nombre d'idéalistes considéraient l'appariement de la poésie et de la philosophie comme lié à un troisième terme, la religion ${ }^{6}$. Les idéalistes s'accordent à dire que la poésie, la philosophie et la religion appartiennent au même champ d'investigation. Mais, alors que Hegel voit l'ordre: poésie, religion, philosophie, les idéalistes semblent parfois inverser l'ordre des deux premiers. Une remarque de nature historique a ici toute son importance: à la fin du dix-neuvième siècle la religion avait aux yeux de certains perdu de sa crédibilité. Nous voyons un aspect de cette question chez Green, dont on peut montrer qu'il cherchait à remplacer la théologie par la philosophie, mais sous un autre angle, dit Jones, on peut voir la poésie comme réussissant là où la théologie a échoué. Dans Browning as a Philosophical and Religious Teacher - notez le titre - il dit que:

De nos jours, nous avons plus que jamais besoin des poètes à [...] des fins éthiques et religieuses. Car les propos du professeur de religion dogmatique ont été considérablement dépouillés de leur ancienne autorité, et le philosophe moral est souvent considéré comme un marchand de lieux communs ou comme le dévot d'une science discréditée. Ils ne sont pas rares ces Anglais cultivés qui trouvent dans les poètes, et dans les poètes seulement, l'expression de leurs convictions les plus profondes touchant à leurs intérêts vitaux les plus profonds (Jones, 1891, 16).

Non seulement il se pourrait que l'art soit supérieur à la religion, mais il se pourrait même (certains idéalistes se le demandaient) qu'il soit aussi supérieur à la philosophie. En réfléchissant sur la manière dont les poètes nous ont appris à voir le divin dans la nature, Mackenzie conclut son Introduction to Social Philosophy par un appel à une nouvelle poésie qui peut nous apprendre à le voir dans l'bumanité et la société. C'est certes quelque chose que la philosophie a déjà mis au jour, mais son argument est que le philosophe en tant

6. «Il n’y a aucune différence entre l'interprétation donnée par la science et celle donnée par la poésie ou la religion, ou encore la philosophie» (Jones, 1891, 192). 
que tel ne peut être ni prophète ni poète. Il ne peut pas rendre sa découverte vivante pour nous. Pringle-Pattison abonde en ce sens et avance que "la religion comme la grande poésie [higher poetry] — simplement parce qu'elles abandonnent toute prétention à une exactitude impossible - nous conduisent, sans aucun doute, plus près de la signification du monde que les formules d'une métaphysique abstraite "(Pringle-Pattison, 1897, 220). Car la philosophie, dans son impitoyable abstraction, ne peut pas combler les attentes de notre vie.

Des réflexions tardives de Bradley peuvent nous aider à comprendre tout cela. Celui-ci jeta certainement un doute sur la position suprême que Hegel accordait à la philosophie: «La philosophie vise la satisfaction intellectuelle, dit-il, elle cherche à prendre possession de la Réalité, mais seulement sous une forme idéale. Et, par conséquent, elle est la réalisation d'un seul aspect de notre être " (Bradley, 1915, 11-12). Car il en est ainsi de tous les aspects de la vie: le plaisir, la moralité, la beauté, le savoir, l'amour sans qu'aucun ne soit perçu comme le bien suprême qui cumule et inclut les autres. Mais on pourrait arguer que la poésie, dans la mesure où elle peut rassembler l'esthétique, l'intellectuel, le moral et le spirituel, est plus vraie pour la vie entière qu'un grand nombre d'autres abstractions partielles.

\section{La vieille querelle entre poésie et philosophie}

Si se tourner vers un précurseur comme Hegel permettait aux idéalistes britanniques de considérer la poésie et la philosophie comme d'importants alliés, cela n'allait pourtant pas de soi. Ils étaient tous formés à l'étude des textes de l'antiquité grecque et latine, et ils savaient donc tous que celui qui fut peut-être le plus grand idéaliste - Platon — était d'un avis différent. Il est intéressant de noter leur réaction, car ils se sentirent tous, presque sans exception, à un moment ou un autre, dans l'obligation de réagir aux positions contraires de Platon.

On se rappellera que, dans la première moitié du livre X de La République, Platon avance que les poètes seront bannis de la cité idéale, puisqu'il «est ancien le conflit entre la philosophie et l'art de la poésie » (République X, $607 \mathrm{~b})$. Il y a deux pommes de discorde. En premier lieu, la poésie est un art d'imitation, mais un art d'imitation peut seulement représenter les choses telles qu'elles apparaissent de l'extérieur. Elle nous donne uniquement la copie d'une copie. Bref, elle génère des faussetés. En second lieu, la poésie encourage de mauvais sentiments. Elle fait appel aux émotions plutôt qu'à la raison et encourage des sentiments dont nous serions normalement honteux et que nous refoulerions. L'art ne devrait seulement dépeindre que ce qui a une valeur. Cependant, Platon conclut en suggérant qu'on pourrait accorder un sursis à la poésie, si elle était en mesure de plaider sa cause.

Il n'y a pas eu de «réponse à Platon» univoque de la part des idéalistes britanniques; son hostilité à l'égard de la poésie a provoqué une variété de réactions différentes, bien que tous fussent gênés par cette manifestation 
d'hostilité. Non seulement celle-ci contredit leurs vues, mais elle est aussi empreinte d'hypocrisie. Comme Nettleship le remarque, "Platon lui-même est à vrai dire un grand poète » (Nettleship, 1897, 354). D'un côté, Mackenzie suggère qu'en tenant des propos aussi absurdes Platon donne délibérément dans l'ironie et l'humour (Mackenzie, 1892, 293). Dans une veine quelque peu similaire, Bosanquet suggère que, plutôt que de condamner l'art comme simplement imitatif et superficiel, nous devrions lire Platon comme reductio de la thèse du genre: "Si la poésie n'est qu'imitation, alors elle n'a aucune valeur et doit être exclue de la société idéale» (Bosanquet, 1904, 30). À l'opposé, A. E. Taylor met en garde contre de telles répliques faciles en insistant sur le sérieux de la critique de Platon et sur le fait que «nous n'apprécierons pas sa position si nous ne comprenons pas clairement qu'il est tout à fait sérieux» (Taylor, 1929, 280).

Les deux idéalistes qui furent les plus près d'accepter la position de Platon furent Edward Caird et Henry Jones. Pour Caird, une résolution de la vieille querelle entre poètes et philosophes dont parle Platon n'est pas plus en vue qu'à son époque. Mais il considère que nous ne devrions pas souhaiter les réconcilier entièrement, car il est préférable (malgré leur étroite relation) qu'elles restent à l'intérieur de leurs limites respectives (Caird, 1892c, 54), un sentiment réitéré par Jones qui recommande vivement que "la querelle [...] dure éternellement» (Jones, 1891, 15). Nettleship soutient plutôt qu'on perd son temps à discuter d'une vraie différence entre philosophie et poésie: "Il n'y a pas de raison de croire qu'un poète ne pourrait pas être véritablement animé, à sa manière, par le même esprit que le philosophe, insiste-t-il, car il y a un lieu, comme Wordsworth l'indique, où la philosophie et la poésie, l'imagination et la science, se rencontrent [...]. Les plus illustres philosophes et les plus grands poètes n'ont pas eu comme règle d'éprouver de l'hostilité les uns envers les autres» (Nettleship, 1897, 354). Entre ces deux extrêmes, une hostilité perpétuelle et une identité harmonieuse, repose la synthèse idéaliste parfaite de J. S. Mackenzie qui soutenait que "c'est simplement parce que la poésie, la religion et la philosophie, dans leur plus haute expression, visent essentiellement la même chose de différentes manières qu'elles sont plutôt aptes à entrer en conflit l'une avec l'autre» (Mackenzie 1917, 17). Peut-être a-t-il vu juste, car il est vrai que frères et sœurs d'une même fratrie et d'une même religion se querellent plus fréquemment et plus violemment que de parfaits étrangers.

James Seth s'accroche au fait que Platon rejette de son État les poètes, non la poésie elle-même, et que cette exclusion n'est pas nécessairement définitive (Seth, 1904-05, 171) ; un point sur lequel insiste aussi Bernard Bosanquet, quand il dit que la suggestion de Platon d'un possible sursis ne devrait pas être rejetée comme simple ironie de sa part (Bosanquet, 1895a, 402). Il y a peut-être deux lignes de pensée ici.

La première vise à limiter la critique de Platon à la littérature de son temps, qui devait avoir atteint une sorte de nadir, et l'estime dans laquelle 
on la tenait. Un certain nombre d'idéalistes ont pris cette voie, puisque cela nous laisse sur une position à laquelle nous pouvons continuer d'adhérer au prix d'un certain snobisme esthétique. Nettleship, par exemple, pense que, dans leurs périodes les moins glorieuses, la poésie et la philosophie semblent être antagonistes, et que nous reconnaissons tous une certaine honte au plaisir que nous avons à lire des romans (Nettleship, 1897, 352 et 354). C'est de nouveau A. E. Taylor qui relève le mensonge attaché à ce type de réponse: "Nous ne faisons que nous jeter de la poudre aux yeux en supposant que Socrate veut seulement réprimer le music-hall bon marché et le mélodrame criard. " Au contraire, dit-il, Platon «propose sérieusement de censurer justement ce que nous considérons être une contribution impérissable d'Athènes à l'art et à la littérature du monde » (Taylor, 1929, 279).

La seconde, peut-être plus typiquement idéaliste, vient de Bosanquet, qui voit la position de Platon comme une des premières étapes, et peut-être une étape inévitable du développement de la compréhension esthétique. Platon avait bien sûr tort de penser que la poésie est simplement l'imitation des apparences visibles, puisqu'à vrai dire elle cherche à dévoiler la structure cachée de la réalité elle-même. Mais, dans la mesure où il offre des critiques nous permettant de dépasser cette première conception simpliste, et dans la mesure où sa propre philosophie exprime une croyance en des Idées cachées, celles-ci étant d'une plus grande réalité que celle des apparences, il nous amène vers la vérité. Dans un style idéaliste classique, mes opposants du passé deviennent des prédécesseurs alliés.

\section{Le style littéraire et la nouvelle philosophie}

On pourrait peut-être se plaindre que tout ce qui a été dit jusqu'ici n'ait été qu'à un méta-niveau, que des discussions abstraites sur la nature de la poésie et de la philosophie. Mais il est à noter que ces vues ont eu un réel impact sur la manière dont les idéalistes britanniques écrivaient sur ces questions. Toute personne ayant lu leurs œuvres ne peut manquer d'en observer le caractère littéraire particulier. Il y a peut-être deux éléments à souligner ici.

Nous remarquons tout d'abord qu'ils citent fréquemment de la poésie. Jetez un coup d'œil dans n'importe quel livre, ou regardez l'index, et vous trouverez un appel constant à la poésie. Nous devrions ajouter ici aussi leur usage fréquent du langage biblique (Jones et Caird en particulier), et leur constante citation d'auteurs comme Carlyle. En outre, tout cela n'est pas simplement décoratif, mais crucial pour la discussion. On emploie la poésie pour avancer des thèses, et pas simplement pour les illustrer.

Notons ensuite la manière même avec laquelle tout un chacun écrit, la nature la plus hautement "poétique » et littéraire de leur langage. Ils étaient tous des écrivains volubiles, utilisant plusieurs pages pour mettre graduellement en place ce que nous appellerions une simple thèse. Parmi les exemples les plus illustratifs qui pourraient être donnés ici, on se contentera de citer un passage à juste titre célèbre de Principles of Logic de Bradley: 
Cela peut provenir d'un vice dans ma métaphysique ou d'une faiblesse de la chair qui persiste à m'aveugler, mais la notion selon laquelle l'existence pourrait être identique à la compréhension paraît aussi froide et fantomatique que le plus ennuyeux matérialisme. Que la gloire de ce monde soit au bout du compte une apparence laisse le monde plus glorieux, si nous l'éprouvons comme le spectacle d'une splendeur encore plus intense; mais le voile des sensations est une déception et une tricherie s'il cache un mouvement sans couleur d'atomes, une trame spectrale d'abstractions impalpables, ou un mystérieux ballet de catégories exsangues. Bien qu'on soit tiré vers de telles conclusions, on ne peut pas les épouser [...]. [Nos principes] ne constituent pas plus le Tout qui commande notre dévotion qu'une dissection de lambeaux humains déchiquetés forment la beauté de cette chair chaleureuse et vivante qui charme tant nos cœurs ${ }^{7}$ (Bradley 1922, 591).

Bien sûr, tous ne furent pas aussi impressionnés. Au fur et à mesure que l'on avançait dans le siècle nouveau, une nouvelle philosophie émergeait avec un nouveau style d'écriture : clair, précis, simple et bref. Usant soit d'un langage ordinaire soit de termes techniques précisément définis, elle était modelée sur le langage des mathématiques et des sciences empiriques. Le bref article scientifique remplaçait les livres écrits sur le mode des tomes des Gifford Lectures. Et les nouveaux écrivains prirent de haut le style de leurs prédécesseurs. Poussée à l'extrême, cette attitude fut celle des positivistes logiques, qui considéraient de tels écrits comme étant littéralement du nonsens. Ayer, dans Language, Truth and Logic, attaqua de manière notoire une phrase "prise au hasard " tirée de Appearance and Reality de Bradley (Ayer, 1971, 49), tandis que Carnap considérait la métaphysique comme une forme de poésie (Carnap, 1995, 178-179); un commentaire qui conduisit Ayer à se moquer des propos de certains philosophes en disant que ces derniers devraient être publiés dans le London Mercury plutôt que dans Mind ${ }^{8}$. Même s'ils n'y voyaient pas qu'un strict non-sens, de nombreux philosophes analytiques des générations suivantes ont considéré les écrits des idéalistes comme difficiles, vagues, confus et rhétoriques. Cette opinion persiste de

7. «It may come from a failure in my metaphysics, or from a weakness of the flesh which continues to blind me, but the notion that existence could be the same as understanding strikes as cold and ghost-like as the dreariest materialism. That the glory of this world in the end is appearance leaves the world more glorious, if we feel it is a show of some fuller splendour; but the sensuous curtain is a deception and a cheat, if it hides some colourless movement of atoms, some spectral woof of impalpable abstractions, or unearthly ballet of bloodless categories. Though dragged to such conclusions, we can not embrace them [...] [Our principles] no more make that Whole which commands our devotion, than some shredded dissection of human tatters is that warm and breathing beauty of flesh which our hearts found delightful. "

8. Le commentaire sarcastique d'Ayer est rapporté dans (Mace, 1934, 38). L'analogie entre la métaphysique et la poésie est délicieusement ironique, étant donné l'estime des idéalistes pour la poésie: il auraient probablement reçu ces critiques de leur métaphysique comme des éloges. 
nos jours; laissons Geoffrey Warnock parler en leur nom : «Il n'est pas surprenant qu'une philosophie de ce genre — si ambitieuse, si délibérément non ordinaire, si résolument profonde - doive se manifester dans une forme d'écriture si particulière. " Mais un philosophe moderne, ajoute-t-il, trouverait ce style "presque insupportable». Bradley, admet-il, était capable, d'une "rhétorique impressionnante, et transmet[tait] dans ses phrases extraordinaires une explosion d'énergie ». Par ailleurs, "la manière de Bosanquet était moins idiosyncratique mais plus littéraire, et proche de la grandiloquence. Il écrivait parfois avec un air de vague sérieux, dans lequel son intention était presque étouffée par le vague du propos. Et dans les écrits d'idéalistes de moindre stature, la solennité et l'absence de clarté semblent souvent s'élever au degré d'une véritable imposture. " Même les arguments de Bradley, suggère-t-il, dépendaient "principalement de la force persuasive et de l'artifice de leur présentation ", de telle sorte qu'en " ôter la parure rhétorique colorée pourrait nuire substantiellement à la doctrine elle-même, voire la laisser sans contenu » (Warnock, 1958, 6-7).

Je veux protester contre ce genre de propos. Comme les philosophes analytiques proposèrent une conception modifiée de la philosophie, ils avancèrent alors un nouveau style littéraire; la clarté et la précision devinrent à l'ordre du jour. Mais c'était injuste de leur part de se retourner contre les idéalistes et de les réprimander sur leur manière d'écrire, alors qu'en fait le style de ces philosophes correspondait tout aussi bien à leur conception de la philosophie. Comme T. S. Eliot l'a dit de la prose de Bradley, c'était "pour ses besoins [...] un style parfait»(Eliot, 1951, 445). Les idéalistes comme les nouveaux philosophes analytiques partageaient l'idée que la conception de la nature propre et de la tâche de la philosophie, et la conception de sa méthode particulière ou de son style étaient deux choses allant de pair: attaquer la seconde n'était rien d'autre qu'une manière mesquine et vaine de signifier son désaccord avec la première. Considérer quelques-unes de ces objections clés et souligner les différences entre les approches philosophiques clarifieront ce point.

1) Pour commencer, il leur a été objecté que leurs écrits étaient trop imprécis et généraux. On pensait qu'avec une portée si large, si tant est qu'ils réussissent à dire quelque chose de vrai, cela ne pourrait être que la plus mince des platitudes. La leçon du principe de vérification fonctionnait ainsi, les mots devant être définis précisément en étant strictement reliés à des applications empiriques.

Mais les idéalistes n'écrivaient pas comme ils le faisaient par accident. Par opposition aux horizons limités des philosophes analytiques, le point de vue philosophique correct était, selon eux, le point de vue global; la philosophie étant précisément la tentative d'observer l'univers, si ce n'est comme un tout, du moins sous l'angle le plus large possible. Pour prendre une analogie artistique, les idéalistes tentaient de peindre de vastes 
tableaux alors que leurs cousins analytiques préféraient travailler sur de petites toiles. Et ils choisirent leurs outils littéraires en conséquence.

Ce sont là des points de vue qui se distinguent par un tempérament sousjacent - dotée d'une opinion pessimiste de nos capacités, l'ambition analytique est modeste et sa vision étroite - , mais également par une théorie du langage sous-jacente. Car l'idée selon laquelle plus les termes sont généraux, moins ils réussissent à exprimer quelque chose n'est que la thèse largement répandue selon laquelle l'intention varie en proportion inverse de l'extension. Ce qui est ici presque un dogme analytique était en fait ouvertement rejeté par des idéalistes tels que Bradley et Bosanquet comme étant soit faux, soit trivial. Selon eux, l'universalité n'était pas abstraite mais concrète, et selon leur logique, plus on élargit, plus on exprime 9 .

2) Une objection du même ordre, mais légèrement différente, consiste à dire que l'écriture des idéalistes était « insaisissable» [slippery]. Leurs arguments se déplacent rapidement de part et d'autre de questions apparemment diverses, sans respect des distinctions et en aboutissant à d'ambitieuses conclusions sur ce qui semble être de fausses identités ou des intuitions vagues quant à la «signification générale des choses » [general drift of things]. Au lieu d'avancer peu à peu, lentement, à petits pas, ils vont trop vite et se hâtent d'aller trop loin, construisant des arguments séduisants mais dont la portée est difficile à saisir avec netteté.

Mais une fois de plus la différence de style reflète une différence plus profonde dans la conception même de ce que c'est que faire de la philosophie. Les philosophes analytiques préconisaient une approche "par étape " [piecemeal], en étudiant les concepts, problèmes et arguments séparément, un à un. À l'opposé, les idéalistes étaient holistes et cherchaient toujours à relier les idées disparates, à trouver les similarités, à atteindre à partir de leur point de départ d'autres domaines. À l'image des poètes qui brillent en associant ensemble des images peu communes et disparates, nous aidant à voir ce que le familier nous a rendu obscur, le philosophe cherche toujours à élargir notre vision et à montrer comment les vérités s'agencent en un ensemble intégré et compréhensif.

3) Une troisième objection contre le style des idéalistes touche son aspect vague et imprécis. Les philosophes analytiques ont prôné la clarté et l'exactitude, déplorant le style nébuleux et flou de leurs prédécesseurs idéalistes. G. E. Moore était particulièrement peiné et exaspéré par leur manque de précision, et un grand nombre de ses articles du début du siècle traitait de sa découverte des ambigüités multiples contenues dans les concepts idéalistes. 
Mais une fois encore, ce qui est présenté comme une maladresse de style est en fait le résultat d'une opinion philosophique profonde et mûrement réfléchie, dans ce cas quant à la nature même de la signification. Car peu d'idéalistes croyaient que les mots ont une signification précise. On devrait selon eux les penser plutôt comme recelant divers niveaux de sens et des implications d'une grande profondeur; des richesses que nous pourrions y trouver ou même qu'ils pourraient déployer pour nous, mais qui certainement s'étendent hors de notre portée, au-delà de l'horizon de notre vision immédiate. La notion selon laquelle il pourrait y avoir une chose telle qu'une analyse unique d'un terme les frappa par son caractère rudimentaire; on devrait plutôt attendre de toute expression non triviale qu'elle contienne plusieurs niveaux d'analyse, s'imbriquant l'un dans l'autre, allant en fin de compte au-delà du terme lui-même. Bradley a expliqué en particulier comment chaque terme ou jugement isole un contenu d'un tout présenté de manière plus large, tout auquel il est alors conditionné, et vers lequel il se tourne inévitablement pour obtenir sa signification complète.

4) Pour évoquer une autre accusation, on a objecté que les idéalistes possédaient une étrange manière de s'exprimer, qu'ils employaient les mots d'une façon mystérieuse. Pour la nouvelle philosophie, la réalité était, par contraste, quelque chose qui devait être décrit dans les termes du langage ordinaire, et le mot d'ordre était «sens commun». Ainsi, par exemple, Moore éprouva une immense difficulté à comprendre ce que Bradley voulait dire lorsque celui-ci affirmait que le temps n'est pas réel, parce qu'il n'employait pas les mots de la «bonne» façon. Selon Moore, une partie de ce que Bradley a cherché à dire doit être - Moore semble parler ici d'autorité — «ce qu'on devrait signifier, c.-à-d. seulement ce que n'importe quelle personne aurait voulu dire en disant que le temps n'était pas réel, et ce qu'une personne ordinaire aurait compris comme ayant été signifié si elle avait entendu ces mots-là »; Bradley emploierait donc ces mots «d'une manière hautement inhabituelle et spéciale » (Moore, 1922, 208).

Mais cette accusation est tout aussi superficielle que les autres. Les idéalistes n'utilisaient pas le type de langage ordinaire que Moore pensait qu'ils auraient dû employer, non par quelque désir pervers de nous embrouiller, mais parce qu'ils ne croyaient tout simplement pas que la réalité puisse être évaluée de cette manière. En premier lieu, le langage ordinaire vient de l'expérience ordinaire et, comme nous l'avons déjà fait remarquer, pour les idéalistes la réalité était quelque chose de caché, quelque chose d'un tout autre genre que l'expérience ordinaire. Mais la question est plus profonde que cela, étant donné qu'ils soutenaient que la réalité n'était pas simplement au-delà de l'expérience, mais aussi au-delà du langage et de la pensée elle-même. Leur philosophie exclut de prendre le lan- 
gage au pied de la lettre. C'est une question importante, permettez-moi donc de l'expliquer plus avant avec deux exemples.

Tout d'abord, nous pouvons considérer T. H. Green. Green est fondamentalement un kantien - sa conscience éternelle est comprise comme celle qui fournit les catégories par lesquelles nous unifions et structurons notre expérience, comme celle qui la rend possible. Mais, tout comme pour Kant avant lui, cela place Green face à un impossible dilemme, car si ce qu'il dit est vrai, alors il ne peut pas le dire. Le problème est que les concepts et les structures s'appliquent seulement à l'expérience et ne peuvent pas légitimement être utilisés hors ou au-delà de l'expérience, pas même pour exprimer les conditions qui rendent cette expérience possible. Ce qui rend la pensée possible ne peut pas lui-même être pensé. Comme le remarque Green: «En parlant de ce principe, nous pouvons seulement utiliser les termes que nous avons; et ces derniers, étant strictement appropriés aux relations ou aux objets déterminés par les relations - que ce principe rend possible mais sous lesquels il ne subsiste pas — sont strictement inappropriés à lui » (Green, 1907, $\mathbb{S}$ 75). Nous rencontrons un problème similaire chez Bradley, pour qui le mode relationnel de la pensée est contradictoire et pointe vers un au-delà de lui-même. Or toute pensée est inévitablement relationnelle, et nous devons donc en conclure que toute pensée pointe au-delà d'elle-même — le «joyeux suicide» de la pensée — vers une réalité absolue, d'une manière certes ressentie, mais indicible (Bradley, 1897, 152).

Le langage est par conséquent limité, et la philosophie travaille à la limite de la signification, à l'extrémité du langage. À cet égard, elle s'apparente à la poésie, car dans un poème la signification ordinaire des mots est elle aussi altérée. C'est pourquoi les idéalistes emploient si fréquemment la poésie; le poète emploie les mots au-delà des limites d'une terminologie abstraite et nous permet d'apprécier des choses qu'on ne peut pas exprimer littéralement. Non seulement le poète peut être philosophe, le vrai philosophe doit aussi être dans une certaine mesure un poète, et par conséquent le même argument explique la raison pour laquelle les idéalistes écrivaient si «poétiquement». Leur exploitation du pouvoir de suggestion des mots aussi bien que leur pouvoir de description étaient parfaitement délibérés.

5) Bien sûr, cet emploi pittoresque du langage provoquait l'accusation de rhétorique, et beaucoup ont pensé depuis que leur écriture était vraiment trop colorée et expressive pour qu'elle puisse être de la philosophie sérieuse. Parfois pesant et solennel, parfois prêcheur et moralisateur, parfois rappelant le sermon du dimanche, le ton de l'idéaliste britannique a rebuté un grand nombre de lecteurs de l'ère edwardienne. C. D. Broad, par exemple, a rejeté avec dédain la philosophie de Green comme n'offrant rien de plus qu' "un arôme apaisant émanant d'un sentiment "d'élévation" 
éthique ", qui transforme les étudiants en personnages arrogants et moralisateurs [prigs] plutôt qu'en philosophes (Broad, 1930, 144). Et ce n'était pas toujours qu'une question de ton, cela devint une question de forme littéraire explicite lorsque Green et les frères Caird écrivirent des sermons ${ }^{10}$.

Ce qui est suggéré ici est tout simplement que de telles façons d'écrire ont quelque chose de peu recommandable et d'amateur. Puisque les positivistes prenaient la science comme modèle, ils visaient une attitude parfaitement rationnelle, supprimant toute valeur et toute émotion de leur discours. De telles choses n'avaient pas leur place, ni en physique ni en philosophie. Mais, alors que les philosophes qui prennent la science pour modèle peuvent se sentir gênés par un langage plus expressif, les idéalistes n'adoptèrent pas ce genre d'approche de la philosophie et virent dans l'attrait des valeurs émotionnelles, morales et spirituelles quelque chose de tout à fait normal. Selon eux, celles-ci constituaient les plus hauts niveaux de l'esprit, et il était donc tout à fait approprié qu'elles soient présentes aux plus hauts niveaux de raisonnement. La nouvelle perspective intellectuelle consistait à soutenir au contraire que de telles valeurs n'ont de place légitime qu'en art, et non en philosophie. Mais pour les idéalistes, le contraste entre la philosophie et l'art n'était pas analogue au contraste entre la raison et l'émotion, mais se rapprochait plutôt du contraste entre la raison et l'imagination - le domaine des sens. En passant de l'art à la philosophie, nous laissons derrière nous ce qui a trait aux sens, mais pas ce qui touche l'aspect moral, émotionnel ou spirituel.

6) Nous arrivons enfin à ce qui est peut-être la plus sérieuse de toutes les objections faites au style des idéalistes britanniques, à savoir que, dans leurs écrits, la poésie, la métaphore et l'art littéraire tiennent lieu d'arguments. Enlevez la rhétorique, dit Warnock, et il ne reste rien. C'est peutêtre une objection plus maladroite que les autres, compte tenu du fait que les idéalistes insistent eux-mêmes sur ce point comme marquant la différence entre philosophie et poésie. Selon eux, la poésie est directe et intuitive, tandis que la philosophie est obtenue par médiation et argumentation. On pourrait presque dire que c'est là sa vertu, puisqu'elle coupe court à toute controverse ${ }^{11}$ ! Mais même ici la situation est plus complexe qu'elle paraît être, comme on peut le voir en se tournant vers l'œuvre de Henry Jones sur Browning.

10. Voir Caird, 1989a; Caird 1898b; Caird, 1907; et Green, 1883. À ceux-ci, nous pourrions ajouter les aphorismes de Bradley (Bradley, 1993).

11. On distingue aussi la poésie de la philosophie par «son caractère spontané et même inconscient» (Caird, 1892c, 59). «La poésie atteint les résultats de la philosophie par des raccourcis et sans user d'une argumentation sans fin» (Jones, 1902, vii). 


\section{Jones sur Browning et le rôle de l'argument}

Le cas de Browning est intéressant. Dans la décade suivant sa mort, en 1889, il fut considéré comme un grand penseur et philosophe. La littérature de cette époque foisonne de titres tels que "Robert Browning, poète religieux" (Symons, 1882), "La philosophie de Browning» (Bury, 1895), "Le théisme de Browning" (Royce, 1897), "Robert Browning, éducateur religieux" (Pigou, 1901), «La poésie et la philosophie de Robert Browning» (Griggs, 1905), «Robert Browning, poète et philosophe» (Sim, 1923). Bien sûr, les temps ont changé, et cette réputation s'est renversée à l'époque du modernisme, sa réputation de penseur profond s'étant complètement effondrée.

Au milieu de cette histoire, en 1891, nous trouvons le livre de Henry Jones Browning as Philosophical and Religious Teacher. On pourrait penser à lire ce titre qu'il ne s'agit que d'un autre livre sur Browning en tant que philosophe, mais en fait le cas est plus complexe. Jones pense en effet de Browning qu'il fut d'une grande importance philosophique, le porte-parole de ce qui pourrait être nommé «l'idéalisme optimiste». Mais Jones insiste pour dire que Browning décevra nos attentes si nous lui faisons l'honneur de le considérer comme un philosophe. Car en vérité il n'était qu'un piètre philosophe, souscrivant à une forme de scepticisme à propos des capacités cognitives humaines, que Jones considérait comme destructeur tant de son optimisme que de son idéalisme. Cette critique était fondée et, par conséquent, paradoxalement, lorsqu'on considère la haute opinion qu'il se faisait du message philosophique de Browning, Jones devint un des principaux acteurs de l'amorce du déclin de la réputation du poète comme penseur.

Cependant, la compréhension que se faisait Jones de la différence entre le message poétique de Browning et ses habiletés philosophiques s'atténua avec le temps, et il envisagea un rapprochement. Dans son livre il présente le problème comme un choc direct de la «tête par rapport au cœur", de la «raison par rapport à l'intuition », mais avec le temps il réalisa que la raison est plus holiste. Elle implique notre conception entière du monde comme êtres humains. Pour cette raison, la conception que se faisait Jones de la méthode propre à la philosophie se rapprocha de celle qu'il se faisait de la poésie. Un texte clé est ici sa conférence de 1905: The Immortality of the Soul in the Poems of Tennyson and Browning (Jones, 1905).

En usant abondamment de la citation, il défend la thèse selon laquelle Browning et Tennyson croient en l'immortalité de l'âme, et que tous deux semblent adopter cette position non par le biais d'arguments, mais par intuition. En fait, déclare Jones, ils se trompent sur eux-mêmes, et leurs positions sont plus solides qu'ils ne le pensent. Loin d'évacuer la raison, ils l'ont pleinement employée. Ils semblent simplement s'appuyer sur de fortes convictions, profondément ressenties, et nous pourrions supposer que la force des convictions vient de la profondeur du sentiment. En réalité la situation est inverse. La puissance du sentiment vient de la force des croyances, et «leur force vient du fait qu'elles ont été unies à notre vie rationnelle par un millier 
de jugements et d'expériences pratiques. Le sentiment de leur force vitale est le résultat d'une intelligence satisfaite, et l'intelligence est satisfaite seulement quand l'expérience prend l'apparence d'un tout harmonieux " (Jones, 1905, 31). Qu'est-ce qui révèle au poète que ce qu'il a à dire est vrai ? Il se peut que le romantique réponde: son cœur. Mais il n'y a pas lieu d'être en désaccord, selon Jones, car "qu'est-ce que "le cœur" dans un tel contexte, si ce n'est l'intégralité de l'expérience rationnelle de l'homme assagi, purifié et éclairé par l'observation exhaustive du vaste ordre des choses et des manières des hommes, et devenu sage par un grand nombre de réflexions» (Jones, 1905, 37). C'est seulement en se basant sur cette prémisse que tous buts, modèles, significations et valeurs peuvent être trouvés dans la vie. Rappelons-nous que ce qui est en jeu ici n'est pas la validité d'un cas quelconque, mais simplement le fait que c'est bien un cas, que ce qui nous est présenté est bel et bien un argument, dans le sens «d'une hypothèse donnant sens à la vie et à l'expérience comme un tout ».

Bien sûr, il n'est pas nécessaire de dire que tout sentiment est de ce type, que tout sentiment n'est qu'un argument d'expérience internalisé, car, comme le dit Edward Caird, "que l'expression “je le ressens" signifie peu ou beaucoup, cela dépend de l'individu qui l'énonce. Il se peut que ce soit l'expression concentrée d'une longue vie de culture et de discipline, ou la voix forte mais vide d'une passion non éduquée et du préjugé » (Caird, 1893b, 160-161). Toutefois, dire que la meilleure poésie n'est pas qu'une simple assertion est quelque chose avec lequel Caird serait certainement d'accord. Dans son essai sur Goethe il avance que, bien que la poésie ne manifeste, ni ne décrive un monde qui s'épuiserait dans son obéissance à la main inerte des lois nécessaires de la nature, il ne devrait certainement pas être supposé que «toute création poétique est produite par une imagination qui suit simplement ses propres rêves et ne se soumet à aucune loi objective» (Caird, 1892c, 58-59). La poésie obéit à ses propres lois, captive d'une nécessité métaphysique plus profonde; l'esprit de la vérité parle à travers le poète et lui fait écrire ce qu'il écrit, et ses vers revêtent un caractère inéluctable et inévitable.

On comprendra mieux ce qui est en jeu ici si on regarde du côté de la théorie idéaliste de l'inférence, telle que développée par Bradley et par Bosanquet. Il se peut que la philosophie soit abstraite, mais dans sa quête de vérité elle revient vers le concret et l'individu. "La vérité est à trouver non pas par l'abstraction mais dans le concret », dit Caird (Caird, 1893a, vol. 1, 151). Ce qui vaut pour le jugement et la vérité vaut également pour l'inférence et la validité: le critère ultime de l'inférence ne repose pas sur un schéma formel abstrait et prédéterminé, mais sur la pleine complexité de la réalité individuelle - un "système» dans les termes de Bosanquet (Bosanquet, 1895b, 40) «l'auto-développement idéal d'un objet» dans les termes de Bradley (Bradley, 1922, 598). 


\section{Conclusion}

Il serait absurde de ma part de conclure cet essai par une affirmation sur la nature réelle de la philosophie, ou sur ce dont traite réellement la poésie, ou sur la vraie relation qui existerait entre elles. Chaque époque jette son propre regard sur le monde, avec son propre caractère, et il n'est pas surprenant que l'Angleterre de la fin de l'époque victorienne ait produit une conception de la philosophie et de la poésie dans laquelle celles-ci sont en parfaite harmonie, comme il est dans l'ordre des choses que les époques suivantes aient envisagé ces deux conceptions de manière nouvelle et différente. Mais à une époque où les philosophes ont peu ou prou à partager avec les poètes, revenir à une conception du monde qui les concevait en plus étroite relation ne peut que nous donner des idées sur la manière dont nous pourrions remanier à notre tour la compréhension de ces deux notions et leur relation.

Traduction de l'anglais par Yaël Joti

\section{Bibliographie}

Ayer, A. J. Language, Truth and Logic, Londres, Penguin Books, 1971.

Bosanquet, B. A Companion to Plato's Republic, Londres, Rivington Percival, 1895a.

- The Essentials of Logic, Londres, MacMillan, 1895b.

- A History of Aesthetic, Londres, Allen \& Unwin, 1904.

- The Principle of Individuality and Value, Londres, Macmillan, 1912.

- Science and Philosophy and other essays, Londres, Allen \& Unwin, 1927.

Bradley, F. H. Appearance and Reality, $2^{\mathrm{e}}$ édition, Oxford, Clarendon Press, 1897.

-. Essays on Truth and Reality, Oxford, Clarendon Press, 1915.

- Aphorisms, dans F. H. Bradley, The Presuppositions of Critical History and Aphorisms, G. Stock (dir.), Bristol, Thoemmes Press, 1993.

- The Principles of Logic, $2^{\mathrm{e}}$ édition, Oxford, Clarendon Press, 1922.

Broad, C. D. Five Types of Ethical Theory, Londres, Routledge \& Kegan Paul, 1930. Bury, J. "Browning's Philosophy », dans E. Berdoe (dir.), Browning Studies, Londres, George Allen, 1985, 28-46.

Caird, E. «Wordsworth», dans Caird (1892d), vol. 1, 1892a, 147-189.

—. "The Problem of Philosophy at the Present Time ", dans Caird (1892d), vol. 1, 1892b, 190-229.

—. "Goethe and Philosophy ", dans Caird (1892d), vol. 1, 1892c, 54-104.

- Essays on Literature and Philosophy, Glasgow, James Maclehose, 1892d.

- The Evolution of Religion, Glasgow, James Maclehose, 1893a.

- The Social Philosophy of Comte, Glasgow, James Maclehose, 1893b.

—. Lay Sermons and Addresses, Glasgow, James Maclehose, 1907.

-. University Addresses, Glasgow, James Maclehose, 1898a.

- University Sermons, Glasgow, James Maclehose, 1898b.

Carnap, R. «Le dépassement de la métaphysique par l'analyse logique du langage », dans A. Soulez (dir.), Manifeste du Cercle de Vienne et autres écrits, Paris, Presses Universitaires de France, 1985, 153-179.

Desmond, W. Art and the Absolute: A Study of Hegel's Aesthetics, Oxford, Clarendon Press, 1986. 


\section{Philosophiques / Printemps 2009}

Eagleton, T. The Meaning of Life, Oxford, Oxford University Press, 2007.

Eliot, T. S. "Francis Herbert Bradley", dans T. S. Eliot, Selected Essays, Londres, Methuen, 1951, 358-368.

Green, T. H. The Witness of God and Faith. Two Lay Sermons, Londres, Longman \& Green, 1883.

- Prolegomena to Ethics, Oxford, Clarendon Press, 1907.

Griggs, E. H. The Poetry and Philosophy of Browning, New York. B. W. Huebsch. Hegel, G. W. F. Hegel's Philosophy of Fine Art, Londres, Kegan Paul, Trench and Trubner, 1905.

—. Cours d'esthétique, 3 vol., Paris, Aubier, 1995-7.

Jones, H. Browning as Philosophical and Religious Teacher, New York, Thomas Nelson, 1891.

- "Introduction », dans P. Janet et G. Séailles, A History of the Problems of Philosophy, volume I, Londres, MacMillan, 1902, vii-xvi.

- The Immortality of the Soul in the Poems of Tennyson and Browning, Londres, Philip Green, 1905.

—. Idealism as a Practical Creed, Glasgow, James Maclehose, 1909.

—. A Faith that Enquires, Londres, Macmillan, 1922.

Mace, C. A. "Representation and Expression", Analysis, vol. 1, 1934, 33-38.

Mackenzie, J. S. An Introduction to Social Philosophy, Londres, Macmillan, 1890.

-. A Manual of Ethics, Londres, W. B. Clive, 1892.

- Elements of Constructive Philosophy, Londres, Allen \& Unwin, 1917.

Mander, W. J. «Bosanquet and the Concrete Universal», The Modern Schoolman, vol. 77, 2000, 293-308.

Moore, G. E. Philosophical Studies, Londres, Kegan Paul, Trench, Trubner, 1922.

Nettleship, R. L. "Lectures on the Republic of Plato", dans R. L. Nettleship, Philosophical Lectures and Remains, volume II, Londres, Macmillan, 1897.

Oakley, H. History and Progress, Londres, Allen \& Unwin, 1923.

Pigou, A. C. Robert Browning as Religious Teacher, Londres, C. J. Clay, 1901.

Pringle-Pattison, A. S. Man's Place in the Cosmos and other Essays, Edimbourg, William Blackwood, 1897.

Royce, J. "Browning's Theism ", dans Boston Browning Society Papers, Londres, MacMillan, 1897, 18-25.

Seth, J. "The Relation of the Ethical to the Aesthetic Element in Literature" International Journal of Ethics, vol. 15, 1904-5, 162-72.

Sim, F. H. Robert Browning Poet and Philosopher, Londres, T. Fisher Unwin, 1923. Symons, A. W. "Robert Browning as a Religious Poet», The Wesleyan-Methodist Magazine, décembre 1882, 1882, 943-947.

Taylor, A. E. Plato. The Man and his Work, Londres, Methuen, 1929.

Warnock, G. J. English Philosophy since 1900, Oxford, Oxford University Press, 1958. 